\title{
Le parapluie de Camille Laurens
}

\author{
Martine Delvaux
}

\begin{abstract}
Camille Laurens presents her novel Dans ces bras-là as a "book on men," a catalogue of masculine characters portrayed by a female narrator. While this operation may appear to be essentializing, a fetishizing of sexual difference, Delvaux's reading shows how, in fact, the novel deconstructs gender, in a manner resembling the deconstructive work that Jacques Derrida finds in Nietzsche.
\end{abstract}

Au début d'Éperons. Les Styles de Nietzsche, Derrida affirme que si le titre retenu pour la séance dans le cadre de laquelle il présente sa conférence est la question du style, c'est plutôt la femme qui sera son sujet. "Il resterait à se demander ", écrit-il, " si cela revient au même - ou à l'autre " (27). Pour que la femme (ou l'homme) soit le sujet d'une communication ou d'un écrit, il faudrait y croire, y trouver quelque chose, une vérité par exemple, un sens, une présence. Et pourtant, ce que Derrida tâche de faire dans Éperons, c'est bien une lecture de la femme comme ce qui se refuse au discours de vérité, à ce que Derrida nomme le " phallogocentrisme ». Si la femme, dans la pensée occidentale, s'est vu attribuer la qualité de la présence (en tant que corps, matière) et donc de la vérité, Derrida la révèle autre, à jamais fuyante, échappant sans cesse à ce qui voudrait la saisir, l'arrêter pour la définir: et celui qui est coupable de cet effort de définition, $c^{\prime}$ est l'homme, en particulier le philosophe dont le logocentrisme s'ancre dans la figure de la femme, qui en fait son fétiche. ${ }^{1}$

Contre cette tendance, Derrida affirme qu' « il n'y a pas d'essence de la femme parce qu'elle écarte et s'écarte d'elle-même. Elle engloutit, envoile par le fond, sans fin, sans fond, toute essentialité, toute identité, toute propriété " (38). De fait, la femme n'est peut-être pas quelque chose, suggère-t-il, et l'homme non plus, même entre les bras de l'écriture, même dans ces bras-là. Car si la femme (chez le Nietzsche de Derrida) est celle qui refuse la castration de la vérité, qui ne croit pas en la vérité, elle est ce qui s'oppose à l'opposition-castration, à ce qui coupe, découpe le sens, le fétichise en y logeant des essences, y compris le sens de ce qu'elle est, de ce qu'ils sont. La femme, elle, jette un voile dessus: 
La 'vérité' ne serait qu'une surface, elle ne deviendrait vérité profonde, crue, désirable que par l'effet $d$ 'un voile: qui tombe sur elle [...] Il suffirait de suspendre le voile ou de le laisser d'une autre façon tomber pour qu'il n'y ait plus de vérité ou seulement la 'vérité' - ainsi écrite. Le voile/tombe. (46)

Le voile met en bière la vérité, l'encrypte et la met en berne. Le voiletombe, et cette tombe, ce voile, est le style. C'est l'abîme, l'écart démultiplié. " Il n'y a pas de vérité de la femme mais c'est parce que cet écart abyssal de la vérité, cette non-vérité est la 'vérité' » (Derrida 38). Cet écart de la vérité " qui s'enlève d'elle-même, qui se lève entre guillemets " (44), c'est celui du style qui écarte en coupant, en inscrivant. La femme jette un voile sur la vérité, tisse un hymen qui suspend le rapport à la castration comme découpure d'une vérité (Derrida 47), comme fétichisation du sens, refusant de dire s'il y a ou non quelque chose là, si quelque chose est disparu, si quelque chose est attendu. " La 'femme' a besoin de l'effet de castration [...] mais évidemment elle n'y croit pas. Est 'femme' ce qui n'y croit pas et qui en joue » (48). Qui joue et met en joue par le biais du style. Car, est-ce qu'une femme pourrait nous retenir, demande Nietzsche, dont on n'imaginerait pas qu'elle sache manier le poignard (dans Derrida 41), c'est-à-dire, bien sûr, le style?

Et le style, ici, c'est elle: Camille Laurens. C'est la forme qu'elle confère à son roman Dans ces bras-là, ce « livre sur les hommes, sur l'amour des hommes » (14). C'est la façon dont elle signe leurs entrées et leurs sorties, ce jeu de va-et-vient qu'est l'ordre et le désordre de leur apparition dans sa vie. Et elle, qui ne serait pas « la femme du livre » mais un personnage «qui ne se dessinerait justement qu'à la lumière des hommes rencontrés » - hommes qu'elle décrit comme « ce jour autour d'elle, ce qui la rend visible, ce qui la crée, peut-être » (15) - elle, c'est le style. Et le style, donc, c'est la femme: «Elle (s')écrit. C'est à elle que revient le style. Plutôt: si le style était [...] l'homme, l'écriture serait la femme » (Derrida 44). Laurens manie le style par l'écriture, cette opération féminine que Derrida trouve chez Nietzsche et qui correspond à la suspension de la vérité (44), à l'attribution de guillemets, à l'aveuglement d'un discours philosophique qui ainsi sombre à sa perte se révélant incapable de vérités, de fétichisations.... L'écriture, aussi, comme le parapluie oublié par Nietzsche (du fragment inédit que Derrida cite: « J'ai oublié mon parapluie »), lisible et recelant un secret, «simul[ant] une vérité cachée dans ses plis » (111). L'écriture, enfin, 
comme des bras ouverts sur une femme qui ne se laisse pas prendre, qui s'offre en s'échappant, ( $s^{\prime}$ )écrit en s'écartant, en dépliant puis en repliant les voiles.

Se dérobant sans cesse sous ses voiles, la femme que l'homme investit de " la félicité et [de] la retraite " (Nietzsche dans Derrida 35), d'un calme qui l'éloigne « de l'ardeur et du ressac » (Nietzsche dans Derrida 33), échappe à la vérité. De fait, sa vérité consiste à échapper à la vérité ellemême, car si, chez Nietzsche, la femme est une figure de la vérité, « elle sait qu'il n'y a pas la vérité, que la vérité n'a pas lieu et qu'on n'a pas la vérité. Elle est femme en tant qu'elle ne croit pas, elle, à la vérité, donc à ce qu'elle est, à ce qu'on croit qu'elle est, que donc elle n'est pas » (40). Elle croit si peu à la vérité, que celle qui la concerne ne lui importe même plus. Ainsi, la vérité, que Derrida assimile à la castration, n’a pas lieu (48). Plutôt, d'y accéder, d'y croire, ferait entrave à l'écriture. Et donc, d'écrire Camille Laurens:

Si on pouvait se nommer, si on savait se présenter dans l'évidence de son sexe, dans la certitude de son être, dans le rayonnement de cette double vérité - moi et l'autre, l'autre et moi - on n'écrirait pas, il n'y aurait pas d'histoire, pas de sujet, pas d'objet. Je n'écrirais pas, si vous étiez l'homme. Je vivrais peut-être. (184, je souligne)

Je suis l'homme. N'est-ce pas merveilleux? Un homme qui s'avance et qui dit: je suis l'homme. Il faudrait pouvoir se tenir en face, rencontrer ses yeux et dire: je suis la femme. (183)

Mais que voudrait dire une telle phrase: je suis la femme, si être la femme est impossible, si la femme n'existe pas? Si, selon Derrida, "c'est l' "homme" qui croit à la vérité de la femme, à la femme-vérité » (50), la femme de Camille Laurens feint de croire à la vérité des hommes, à la véritéfétichiste; elle fait semblant d'être la femme. Elle fait semblant d'avoir un trou. «J'ai un trou » (Laurens 218), écrit la narratrice pour indiquer qu'elle a un blanc, que ce qu'elle voulait dire à son psychanalyste « sur la différence entre les hommes et moi ", sur cette différence qui est là malgré tout, même à l'extérieur du conflit, elle l'a oublié: " je voulais vous dire quelque chose là-dessus " (217). Trou de mémoire, absence $d u$ sexe qui est le sexe même, le sexe du même tout en étant aussi, déjà, celui de l'autre, celui qu'elle a: 
Parce qu'au fond, assez souvent j'ai l'impression d'être un homme - je veux dire: je ne me sens pas féminine au sens classique du terme; plutôt virile, en fait (...) Mais ce n'était pas ce que je voulais dire, je sais que non. Ah! C'est tout près et pourtant impossible à saisir, je n'y arrive pas. Non, vraiment non: c'est perdu, ça ne me reviendra pas. J'ai un trou. (218)

Un trou, un oubli, comme dans le fragment que Derrida cite de Nietzsche: "J'ai oublié mon parapluie », un oubli qui n'en est pas vraiment un, un oubli qui s'oublie en tant qu'oubli. C'est lui qui fait dire à Derrida que le style, il ne faut pas l'oublier, c'est le parapluie, l'objet « éperonnant, l'objet long, oblong, arme de parade autant qu'il perfore, la pointe oblongifoliée tenant sa puissance apotropaïque des tissus, toiles, voiles qui se bandent, se ploient ou déploient autour d'elle » (32). ${ }^{2}$

Le parapluie, objet double, à la fois éperon et voiles, fétiche ici de l'opération féminine. Car la femme, selon Derrida, ne se trouve pas inscrite en tant que telle chez Nietzsche. Plutôt, elle est la non-essentialité et la fluidité sans bornes du graphème (Argyros 30), d'un graphème semblable à la phrase « j'ai oublié mon parapluie » qui, comme la femme, est contingente et dont le sens est nécessairement instable. Fragment nietzschéen inédit, ces mots ne peuvent être saisis à l'intérieur d'un horizon délimité, ne peuvent être interprétés de façon monologique (Argyros 30). Marquée par la notion de l'oubli du parapluie plutôt que par son essence en tant que parapluie, cette phrase, comme la femme, représente l'opération de l'écriture; elle est l'écriture qui énonce alors même qu'elle perturbe, qui affirme en même tant qu'elle oublie. Il ne faut pas oublier que l'oubli est constitutif de la signification, qu'il la hante, qu'il y laisse sa trace. L'oubli marque la différance. Il ne faut pas oublier que le parapluie est un objet qu'on oublie, objet dont on oublie qu'il est à la fois voile et éperon, voiles pliées-dépliées sur un éperon érigé-affaissé.

Il en est ainsi de Camille Laurens et de son trou, de ce trou-oubli qu'elle oublie; Laurens, la voilée dévoilée, la castrée non-castrée, celle qui feint d'avoir oublié son parapluie alors même qu'elle le tient, alors qu'elle sait qu'elle ne se fera pas mouiller. « Je ne serais pas la femme du livre » écritelle, "Ce serait un roman, ce serait un personnage » (15) et de fait, elles sont toujours deux, la narratrice double et dédoublée, à la fois je et elle, mais surtout, à la fois elle et lui: « Sujet: moi. Je suis pleine d'hommes, voilà le sujet » (182). Sujet et objet. Voiles suspendues et transpercées. Femme- 
parapluie, donc: Camille au nom double, ${ }^{3}$ androgyne, est la femme-tain dans laquelle ils se mirent, la femme-papier dans laquelle ils viennent s'imprimer. Car ces hommes qui mettent la narratrice en lumière, qui la révèlent, qui la font naître belle comme le jour, s'ils se reflètent en elle, c'est toutefois elle qui les regarde, elle qui se remplit de leur image " comme un lac du reflet d'un ciel », « les maintient d'abord dans cette distance qui permet de les réfléchir » (17). C'est elle qui regarde l'autre sexe, le fixe, le dépeint, l'écrit. C'est elle qui porte le sexe opposé.

Elle les voit toujours comme ces voyageurs assis vis-à-vis d'elle dans les trains maintenant rares où cette disposition existe encore: non pas à côté d'elle, dans le même sens, mais en face, de l'autre côté de la tablette où gît le livre qu'elle écrit. Ils se tiennent là. C'est le sexe opposé. (17)

L'homme, c'est celui qui n'est " pas son genre, non. Le genre qu'elle n'a pas » (92), puisqu'elle, ce qu'elle a, c'est un trou, un genre manqué, un parapluie oublié. On la dirait castrée. Et pourtant. On dirait qu'elle fait semblant. « J'ai un trou » dit-elle. Mais j'ai aussi autre chose: «Il y a un homme dans mes yeux » (287). Car si les hommes se tiennent en face du livre à venir, de l'autre côté de l'écriture, en opposition; s'ils sont « la rive étrangère » (92), l'homme, chez Laurens, c'est aussi la mer qui permet l'écriture (contre la mère dans laquelle elle se trouve déjà: « ma mère, c'est moi. Je suis dedans » [144]), l'eau sombre où elle jette son ancre: «Il est à côté, je suis à côté, il y a une distance, une différence, un espace entre nous, infranchissable, c'est pourquoi j'en parle, pour combler, pour me rapprocher. Infranchissable, il paraît: un abîme. J'en parle pour le mesurer, pour y tomber peut-être " (144). C'est elle le livre qu'elle écrit sur eux.

Mais l'homme, c'est aussi le trou, comme en témoigne la suite d'amants manchots qui défilent au fil de la vie de la narratrice de Dans ces bras-là. Bras qui ne peuvent que s'ouvrir sur une absence. Main absente qui tisse le lien avec celle qui a un trou. « Elle ne sait dire ce qui justifie [...] le retour de l'homme sans bras, et de quelle part manquante se nourrit son désir [...] L'amour naît-il de ce qu'il y a là quelque chose d'impossible? L'amour estil ce qu'on n'embrasse jamais que du regard? » (78) Et tout contre l'homme dont le bras est manquant, elle manipule le style, elle écrit pour lui, à sa place, un livre qui vient combler le trou, l'absence du bras. C'est aussi dans ces termes que Laurens présente l'écriture dans Philippe (1995), le livre 
portant sur son fils mort à la naissance: « Faire un livre, faire l'amour: effort vain d'abolir l'intervalle. Écrire: mettre des mots dans le trou, colmater » (19). L'homme, c'est aussi cet abîme de l'écriture que la narratrice explore dans la distance: «Elle cherche ce qui fait d'eux des hommes, elle tourne autour de ce point » (242). Elle danse. «Valse à deux temps » (19). Chorégraphie.

Chez Laurens, l'écriture et la lecture entraînent la femme et l'homme à l'intérieur d'une danse qui à la fois les joint et les sépare: «Un homme et une femme qui dansent, est-ce que c'est un couple, pour vous? Une union susceptible de parvenir à l'unité? Pour moi, ça fait deux » (73). Ils sont à la fois un et deux: « il ne tient qu'à vous d'être moi, il ne tient qu'à moi d'être vous - un seul corps de nos deux corps seuls » (288). Mouvement vers, frôlement, caresse qui constitue un abord mais non une dissolution de l'autre, proximité levinassienne qui doit préserver l'altérité. L'écriture est une danse où les corps se révèlent en même temps soudés et scindés: « Me rapprocher de l'homme, voilà l'objectif. Mais pas au point d'espérer le saisir ou le rejoindre. Une danse, tout au plus. Un tour de valse, avec du jour entre les corps » (73). Du jour entre les corps, le maintient de l'abîme entre eux, de ce qui marque la différence.

Car la distance doit demeurer. La distance dont la femme, chez Nietzsche, est caractérisée, distance qui constitue sa qualité même (car elle n'est peutêtre pas autre chose, quelque chose d'autre que cette distance, " l'abîme de la distance [...], la distance elle-même » [38]), sa force de séduction, son pouvoir. Il en est de même içi de l'exercice de l'écriture et de la lecture qui, si elles se présentent comme le lieu de la rencontre entre hommes et femme, font néanmoins abîme, traçant le jour entre les corps, inscrivant la distance. Le style ouvre une brêche, creuse un trou qui signe " une opération éperonnante plus puissante que tout contenu, toute thèse et tout sens » (Derrida 86). Le style, « de son éperon prot[ège] contre la menace terrifiante, aveuglante et mortelle (de ce) qui se présente, se donne à voir avec entêtement: la présence, donc, le contenu, la chose même, le sens, la vérité [...] » (Derrida 30).

De fait, si Camille Laurens semble prendre le parti de l'essentialisme opposant hommes et femmes à l'intérieur de ce qui se donne comme un discours, castrant et castré, de vérité - en fait elle n'y croit pas. Comme la femme, selon Derrida, ne croit pas à la castration: « la 'femme' a besoin de l'effet de castration ", écrit-il, 
sans lequel elle ne saurait séduire ni ouvrir le désir - mais évidemment elle n'y croit pas. Est 'femme' ce qui n'y croit pas et qui en joue. En joue: d'un nouveau concept ou d'une nouvelle structure de la croyance visant à rire. De l'homme - elle sait, d'un savoir auquel aucune philosophie dogmatique ou crédule n'aura pu se mesurer, que la castration $n^{\prime}$ a pas lieu. (48)

Et malgré les apparences, $\mathrm{l}^{\prime}$ « homme » n'aura peut-être pas été le sujet de Camille Laurens ${ }^{4}$ qui l'aura, peut-être, oublié. Comme l'inédit de Nietzsche ( "J'ai oublié mon parapluie »), il faudrait lire dans ce roman «ce pour quoi il se donne en se dérobant, comme une femme ou une écriture » (Derrida 108). Il faudrait le lire en perforant le voile qui lui donne pudiquement l'apparence de la construction d'un sens, $d u$ sens, celui de l'homme et de la femme et de leur différence. Ici, l'homme n'a pas lieu (ni la femme); il est (ils sont) un effet de style, le tracé d'un abîme lisible et illisible, révélation et secret « non qu'il détienne un secret mais parce qu'il peut toujours en manquer et simuler une vérité cachée dans ses plis » (111).

\section{Notes}

${ }^{1}$ Dans son article « The Seduction of Sirens: Derrida and Woman », Wendy O'Shea-Meddour s'attarde à la figure de la sirène qui hante à la fois le texte de Nietzsche et celui de Derrida: «Although Homer's sirens demand an exchange (knowledge for death), they do represent the site of truth. Derrida's new version of the siren in Spurs makes no such claim. Rather, it "is the 'man' who has decided that his discourse on woman or truth might be of any concern to her... For it is man who believes in the truth of woman." It becomes increasingly clear that the siren's powers of seduction depend upon man's willingness to believe in his own discourse on woman (one that asserts that woman is the site of presence) » (479).

2 Dans Jacques Derrida, Geoffrey Bennington écrit: «Dans la mesure où toute trace est trace de trace, aucun texte n'est assez "lui-même" pour se passer du contexte; mais du même coup aucun contexte ne peut vraiment se clore, et on lira indéfiniment une phrase comme "J'ai oublié mon parapluie" sans jamais en venir à bout » (88). 
${ }^{3}$ Il faut noter que Camille Laurens est le pseudonyme de Laurence RuelMézières, pseudonyme qui juxtapose un prénom androgyne et un nom féminin dont la féminité a été en quelque sorte effacée.

${ }^{4}$ Comme l'écrit Derrida dans Eperons: "Il n'y a pas de don de l'être à partir duquel quelque chose comme un don déterminé (du sujet, du corps, du sexe et autres choses semblables - la femme n'aura donc pas été mon sujet) se laisse appréhender et mettre en opposition » (100).

\section{Ouvrages cités}

Argyros, Alexander. " Daughters of the Desert ». Diacritics 10.3 (1980): 2735.

Bennington, Geoffrey et Jacques Derrida. Jacques Derrida. Paris: Seuil, 1991.

Derrida, Jacques. Eperons. Les Styles de Nietzsche. Paris: Champs / Flammarion, 1972.

Derrida, Jacques et Christie V. McDonald. « Choreographies ». Diacritics 12.2 (1982): 66-76.

Laurens, Camille. Dans ces bras-là. Paris: P.O.L., 2000.

—. Philippe. Paris: P.O.L., 1995.

O'Shea-Meddour, Wendy. "The Seduction of Sirens: Derrida and Woman ». Textual Practice 13.3 (1999): 465-86. 